\title{
Geometry and the Simplex: Results, Questions and Ideas
}

Karim Adiprasito (University of Copenhagen, Denmark and Hebrew University of Jerusalem, Israel)

When we try to understand an object, first we have to describe it. And for many objects, the best description, in the sense of what we can do with it, is a combinatorial one.

What has always fascinated me is how little we know about such combinatorial descriptions, up close. One case that often arouses interest is a triangulation; a simplicial complex that models a topological or algebraic object.

Let me explain what I mean: we know pretty well what to do with a triangulated manifold if we do not care about the triangulation in question, that is, if we are allowed to retriangulate. Then we are really interested in the global, topological properties rather than the combinatorial ones. Consider instead, for example, the question of counting the number of vertices in a triangulation. The issue arises: this is an invariant of the underlying simplicial chain complex that is not recognised by the homology.

This is often in contrast to geometry, where we understand the local-to-global correspondence in a much better way (though it is far from perfect). This goes so far that even some of the interesting results on triangulated manifolds are proven using a geometric detour, such as Cheeger's trick of seeing a triangulated manifold as a smooth one with singular points [Che86].

That is not to say that smooth questions are easy, but the technology we have, in the form of differential geometry, is developed much further. So we pay a heavy price for the fact that we can actually work with these spaces, encode them, and are still trying to make many of the smooth calculations work in the discrete setting, such as computing characteristic classes [GM92].

I will attempt in this note to discuss some of the techniques that are used to understand such combinatorial-geometric problems, and will start with an algebrao-geometric technique.

\section{Complexity of embeddings}

Consider the following combinatorial question: We are given a simplicial complex $\Delta$ that we know embeds into the euclidean space $\mathbb{R}^{d}$. How complicated can $\Delta$ be? That is, for instance, how many faces of dimension $k$ can it have, given the number of faces of dimension $i<k$ ?

If we, for example, embed into dimension $\mathbb{R}^{2}$, then the answer is classical, and Descartes or Euler have already answered it: a planar graph without double edges or loops can have at most three times as many edges $E$ (1-dimensional simplices) as it has vertices $V$, i.e. we have the famous inequality

$$
E \leq 3 V
$$

What about, say, complexes of dimension two and higher? This is an important question by Grünbaum from 1967, later refined by Kalai and Sarkaria [Grü03, Kal91].
The interesting question then is to embed into dimension 4. Well, we know in principle what to do: we could attempt to use the van Kampen obstruction and topological techniques. However, these techniques often see mostly the topology of the complex, not its combinatorics. That makes it supremely difficult to extract a good bound, and it means that even if Wagner's theorem extends in some sense, it is difficult to tickle it enough to get a practical bound from those versions, such as [Nev07].

A second fact that helps in the planar setting: we can always extend a planar graph that is large enough (at least 3 vertices) to a triangulation of the plane without adding any edges. The same is not possible in higher dimensions. So, an entirely new trick is needed. A key result of [Adi18] is as follows:

Theorem 1.1. Given a simplicial complex $\Delta$ that piecewiselinearly embeds in $\mathbb{R}^{4}$, then

$$
T \leq 4 E,
$$

that is, the number of triangles $T$ (2-dimensional simplices) exceeds the number of edges $E$ (1-dimensional faces) by a factor of at most 4 .

This result is asymptotically tight, though it can be improved to a tight result by appending an additive error term [Adi18, Remark 4.9]. The result extends similarly to higher dimensions (with a similar bound depending linearly on the embedding dimension), but several questions, including the main one, remain open:

Problem 1.2. Does the result extend to topological embeddings? Does it extend to cell complexes that are strongly regular, that is cell complexes whose partially ordered set of faces is an atomistic lattice?

The best result towards topological embeddings is due to Parsa [Par18], who proved that

$$
T \leq c E V^{\frac{1}{3}}
$$

where $V$ is the number of vertices. It seems that we have to develop some new techniques for the topological case.

To prove the bound of Theorem 1.1, the idea is to move away from simplicial homology, and instead consider a topological model where points itself generate meaningful topology. This is, topologically, encoded in the notion of momentangle complexes, a space built out of replacing the simplices of the complex with tori of different dimensions [BP15]. Rather than work with these torus complexes, it is more convenient to think about it in algebraic terms. So we follow Melvin Hochster's idea, see [Sta87], modelled after Chow rings of toric varieties. 


\section{$2 \quad$ Face rings}

If $\Delta$ is an abstract simplicial complex defined on the groundset $[n]:=\{1, \cdots, n\}$, let $I_{\Delta}:=\left\langle\mathbf{x}^{\mathbf{a}}: \operatorname{supp}(\mathbf{a}) \notin \Delta\right\rangle$ denote the nonface ideal in $\mathbb{R}[\mathbf{x}]$, where $\mathbb{R}[\mathbf{x}]=\mathbb{R}\left[x_{1}, \cdots, x_{n}\right]$. Let $\mathbb{R}^{*}[\Delta]:=\mathbb{R}[\mathbf{x}] / I_{\Delta}$ denote the face ring of $\Delta$. Now, we pick a sufficient number of linear forms to make sure the quotient is finite dimensional:

The reduced face ring with respect to such a system $\Theta$ is

$$
\mathcal{A}^{*}(\Delta):=\mathbb{R}^{*}[\Delta] / \Theta \mathbb{R}^{*}[\Delta]
$$

The following theorem summarises observations by Hochster, Reisner and Stanley:

Theorem 2.1 (Face numbers and Poincaré duality). For a triangulated sphere $\Sigma$ of dimension $(d-1)$,

$$
h_{i}(\Sigma)=\operatorname{dim} \mathcal{A}^{i}(\Sigma) .
$$

Moreover, the pairing

$$
\mathcal{A}^{i}(\Sigma) \times \mathcal{A}^{d-i}(\Sigma) \rightarrow \mathcal{A}^{d}(\Sigma) \cong \mathbb{R}
$$

is perfect.

Here,

$$
h_{k}:=\sum_{i=0}^{k}(-1)^{k-i}\left(\begin{array}{l}
d-i \\
k-i
\end{array}\right) f_{i-1},
$$

where $f_{j}$ denotes the number of faces of dimension $j$ in a simplicial complex.

Now, to return to our original question: If the embedding of $\Delta$ is piecewise-linear, then it is not hard to see (and proved for instance in [Bin83]) that $\Delta$ extends to a triangulation of a piecewise-linear sphere $\Sigma$ of dimension $2 k$.

It is now not hard to notice that the quotient $\mathcal{A}^{*}(\Delta)=$ $\mathcal{A}^{*}(\Sigma) / I_{\Delta}$ satisfies

$$
\operatorname{dim} \mathcal{A}^{k}(\Delta) \geq f_{k-1}(\Delta)
$$

and

$$
\operatorname{dim} \mathcal{A}^{k+1}(\Delta) \leq f_{k}(\Delta)-(k+1) f_{k-1}(\Delta)
$$

Notice further that

$$
\mathcal{A}^{k}(\Sigma) \cong \mathcal{A}^{k+1}(\Sigma)
$$

by the Poincare duality property above.

It then remains to establish the inequality

$$
\operatorname{dim} \mathcal{A}^{k}(\Delta) \geq \operatorname{dim} \mathcal{A}^{k+1}(\Delta)
$$

\section{$3 \quad$ Biased pairing properties and Lefschetz}

Here are two critical observations concerning the above inequality.

- It follows from the Lefschetz property, i.e. the isomorphism

$$
\mathcal{A}^{k}(\Sigma) \stackrel{\cdot \ell}{\rightarrow} \mathcal{A}^{k+1}(\Sigma)
$$

induced by multiplication of some element $\ell$ in $\mathcal{A}^{1}(\Sigma)$. This is an important, and difficult to prove property from algebraic geometry [Laz04], and is known in the case that $\Sigma$ is the boundary of a polytope. The desired inequality (1) follows from

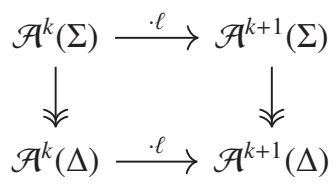

- It follows from the biased pairing property. Consider the kernel $I$ of the map $\mathcal{A}(\Sigma) \rightarrow \mathcal{A}(\Delta)$. Then the desired inequality (1) follows from saying that

$$
I^{k} \times I^{k+1} \rightarrow \mathcal{A}^{d}(\Sigma) \cong \mathbb{R}
$$

is nondegenerate in the first factor. It is a little tricky to give context for this property, it does seem to have been used before.

Let us put these properties into a tiny bit of context.

\section{Interlude: The classical and non-classical Lefschetz theorems}

If $\Sigma=\partial P$, where $P$ is a $d$-dimensional polytope, and one takes $\Theta$ to be the linear system induced by the coordinates of $P$, and $\ell$ is the sum of variables, then $\mathcal{A}(\Sigma)$ satisfies the Lefschetz property with respect to $\ell$. Moreover, if we consider the Hodge-Riemann bilinear form

$$
\begin{aligned}
& \mathrm{Q}_{\ell, k}: \quad \mathcal{A}^{k}(\Sigma) \quad \times \quad \mathcal{A}^{k}(\Sigma) \quad \longrightarrow \quad \mathcal{A}^{d}(\Sigma) \cong \mathbb{R} \\
& a \quad b \quad \longmapsto \quad \operatorname{deg}\left(a b \ell^{d-2 k}\right)
\end{aligned}
$$

then it is definite of sign $(-1)^{k}$ on the kernel

$$
\operatorname{ker}\left[\mathcal{A}(\Sigma)^{k} \stackrel{\cdot \ell^{d-2 k+1}}{\longrightarrow} \mathcal{A}(P)^{d-k+1}\right] .
$$

These are the so-called Hodge-Riemann relations.

Unfortunately, most spheres $\Sigma$ do not arise as boundaries of convex polytopes [Alo86, GP86]. And convexity is crucial: the proof here follows an idea by McMullen [McM96], and the current wave of combinatorial Lefschetz theorems in Coxeter groups [EW14] or matroids [AHK18] all use his basic but amazing idea.

The idea in [Adi18] is different: I discuss what happens for triangulations of general spheres, where Hodge-Riemann relations fail, and instead turn into the so-called Hall-Laman relations, which signify the non-degeneracy of the HodgeRiemann form on subspaces cut out by squarefree monomial ideals, that is, exactly the ideals arising as kernels of maps

$$
I(\Sigma, \Delta):=\mathcal{A}(\Sigma) \longrightarrow \mathcal{A}(\Delta) .
$$

Let me try to give an overview of the ideas:

\section{$5 \quad$ Back to biased pairings}

Now, there are several critical observations that relate the biased pairing property (for all squarefree monomial ideals) and the Lefschetz property, setting up a way to prove the Lefschetz property inductively. Some of the central observations are that Lower dimensional Lefschetz implies biased pairing, and that biased pairing proves Lefschetz. For the first, the following proposition provides a glimpse

Proposition 5.1. Assume $\Delta$ is a rational hypersurface sphere in a sphere $\Sigma$ of dimension $2 k-1=d$. Then $\mathcal{A}(\Sigma)$ satisfies biased Poincaré duality in degree $k$ and with respect to $I(\Sigma, \Delta)$ if and only if

$$
\mathcal{A}^{k}(\Delta)=0 \text {. }
$$

Note that $\mathcal{A}_{k}(\Delta)=0$ is a Lefschetz property: forget one of the elements of the linear system of parameters $\Theta$ of $\mathcal{A}(\Sigma)$, 
arrive at a new and shorter system $\Theta^{\prime}$ and an additional element $\vartheta$. Then the second property is equivalent to the Lefschetz isomorphism for

$$
\mathbb{R}^{k-1}[\Delta] /\left\langle\Theta^{\prime}\right\rangle \stackrel{\cdot \vartheta}{\longrightarrow} \mathbb{R}^{k}[\Delta] /\left\langle\Theta^{\prime}\right\rangle .
$$

Second, we have the following rather beautiful lemma, essentially due to Kronecker:

Lemma 5.2. Given two linear maps

$$
A, B: X \longrightarrow Y
$$

of two vector spaces $\mathcal{X}$ and $\mathcal{Y}$ over $\mathbb{R}$ (or any infinite field). Assume that

$$
B(\operatorname{ker} A) \cap \operatorname{im} A=0 \subset \mathcal{Y} .
$$

Then a generic linear combination $A$ " $+" B$ of $A$ and $B$ has kernel

$$
\operatorname{ker}(A “+" B)=\operatorname{ker} A \cap \operatorname{ker} B .
$$

The connection to the classical Hall matching theorem, which constructs stable matchings in a discrete setting [Hal35]. This lemma is designed to do the same in the setting of linear maps. The idea is now to prove the following transversal prime property: for $W$ a set of vertices in $\Sigma$ if

$$
\text { ker “ } \sum_{v \in W} " x_{v}=\bigcap_{v \in W} \operatorname{ker} x_{v}
$$

Note: proving the transversal prime property for all vertices together is equivalent to the Lefschetz isomorphism

$$
\mathcal{X}=\mathcal{A}^{k}(\Sigma) \stackrel{\bullet \ell}{\rightarrow} \mathcal{Y}=\mathcal{A}^{k+1}(\Sigma)
$$

for $\ell$ the generic linear combination over all variables. This is because

$$
\bigcap_{v \text { vertex of } \Sigma} \operatorname{ker} x_{v}=0
$$

because of Poincaré duality.

Note further that, to see how the biased pairing property implies the transversal property by induction on the size of the set $W$, when we try to apply the criterion by multiplying with a new variable $x_{v}$, adding a vertex $v$ to the set $W$, then we are really pulling back to a principal ideal $\left\langle x_{v}\right\rangle$ in $\mathcal{A}(\Sigma)$, and being asked to prove that $x_{v}$ ker " $\sum_{v \in W}$ " and im " $\sum_{v \in W}$ " $\cap\left\langle x_{v}\right\rangle$ intersect only in 0 .

Note finally that both spaces are orthogonal complements. This is the case if and only if the Poincare pairing is perfect when restricted to either (or equivalently both) of them.

That closes the circle, and gives us a glimpse of the ideas in [Adi18], though the proof takes a detour we do not go over here. We refer the reader to the more friendly introduction [Adi19] to get a better idea.

\section{Some relations}

Let me mention two interesting applications of the individual results above:

\section{Spaces of low rank tensors and the potential for} a trivial lemma

First, let me note that Lemma 5.2, while it seems trivial, has some quite interesting consequences. It tells us that spaces of low-rank maps are restricted. For instance: Consider a space
$L$ of tensors in $V_{1} \otimes V_{2} \otimes \cdots \otimes V_{n}$, where $V_{i}$ are vector spaces over some infinite field.

Define $r(L)$ to be minimum

$$
\operatorname{dim} V_{1}^{\prime}+\operatorname{dim} V_{2}^{\prime}+\ldots
$$

so that $L$ lies in subspace

$$
V_{1}^{\prime} \otimes V_{2} \otimes \cdots \otimes V_{n}+V_{1} \otimes V_{2}^{\prime} \otimes \cdots \otimes V_{n}+
$$

With David Kazhdan and Tamar Ziegler we proved recently that

$$
\mathrm{r}(L) \leq C_{n} \max _{\ell \in L} \mathrm{r}(\ell)
$$

This in particular implies that Schmidt rank is linearly bounded from above by Gowers-Wolf analytic rank for cubics, notions important in analytic combinatorics, see for instance [Lov19]. Indeed, it seems to be a powerful trick to construct high-rank linear maps, and I think Kronecker's lemma might see some interesting further use down the line, in particular to construct Lefschetz isomorphisms in non-algebraic settings (for example [Ven17]). See also [Gur02] for a connection to quantum matchings.

\section{A relation to the Dodziuk-Singer conjecture}

Let us also take a moment to discuss an interesting relation of Proposition 5.1 to the Dodziuk-Singer conjecture [Dod79], alleging that the $\ell^{2}$-cohomology of the universal cover $\widetilde{M}$ of an aspherical $d$-manifold $M$ has vanishing $\ell^{2}$-cohomology, except possibly in the middle dimension.

Assume now that $M$ is triangulated. A central result of [Adi18] is that

$$
\operatorname{ker}\left[\mathcal{A}^{i}(M) \longrightarrow \bigoplus_{v \text { vertex of } M} \mathcal{A}^{i}\left(\mathrm{st}_{v} M\right)\right] \cong\left(H^{i-1}(M)\right)^{\left(\begin{array}{c}
d \\
i
\end{array}\right)}
$$

where st denotes the closed neighbourhood of a vertex. This extends to $\ell^{2}$-cohomology, and with Proposition 5.1 it follows that if $D_{i}$ is a family of compact disks exhausting $\widetilde{M}$, then the Dodziuk-Singer conjecture is true for $\widetilde{M}$ if

$$
\mathbb{R}^{k-1}\left[\partial D_{i}\right] /\left\langle\Theta^{\prime}\right\rangle \stackrel{\vartheta \vartheta}{\longrightarrow} \mathbb{R}^{k}\left[\partial D_{i}\right] /\left\langle\Theta^{\prime}\right\rangle .
$$

are isomorphisms and uniformly bounded as operators.

In some situations, such as the case of right-angled Coxeter groups, it is possible to then define a limit of the rings $\mathbb{R}^{k-1}\left[\partial D_{i}\right]$, with individual elements of the sequence connected by pullback maps. This then leads us to a Hilbert space with a graded algebra structure inherited from the $\mathbb{R}^{k-1}\left[\partial D_{i}\right]$, which we need to establish a Lefschetz property on. It seems promising to understand in this context the relation between the work by McMullen [McM96] and Alesker [Ale03] on Lefschetz theorems for valuations, which also stand in a slightly indirect limit relation to each other.

\section{How small can you make a combinatorial space, then?}

Now you have had a taste of what I am interested in: understanding combinatorial questions in a new algebraic and geometric light, reformulating them, and then proving interesting algebraic and geometric theorems using combinatorial means. We have seen some relations to the Lefschetz property, but I want to change directions and discuss questions 
where a closer understanding of a (discrete) differential geometry seems to be interesting, though we come full circle in the end.

A central problem is often how large a given manifold has to be. This requires specification of what I want to be large or small. One way that low-dimensional topologists like to go, for instance, is to ask for volume and specify a geometric structure (e.g., hyperbolic). Volume, of course, makes sense in the context of bounded geometry, see for instance [CDMW18] for some recent deep results.

Another can be the number of faces a minimal triangulation must have. These measures are tangentially related, but should not be confused with Gromov's notion of simplicial volume [Gro82], which is asking about cell decompositions rather than triangulations.

About the latter, we know embarassingly little. Let us just ask for the minimal number of vertices, that is, how many vertices you need to triangulate a given topological space (assuming you can).

We do know what, for example, the smallest triangulation of a ball is, or what the smallest triangulation of the sphere is (hint: it is the simplex and its boundary.)

We also know that the number of vertices cannot be lower than the ball-category (the number of disks needed to cover a manifold), or the more studied Lusternik-Schnirelmann category (the number of contractible sets needed to cover a manifold) [CLOT03]. In particular it is bounded from below in terms of the cup length of the space in question. In fact, it is easy to show, and observed by Arnoux and Marin, that for a space of cup length $n$, one needs $\left(\begin{array}{c}n+2 \\ 2\end{array}\right)$ vertices [AM91].

Satoshi Murai also gave a lower bound in terms of the Betti numbers of (closed and orientable) manifolds [Mur15], which was simplified and generalised to general manifolds by Adiprasito and Yashfe [Adi18, AY20]. Essentially, we have that if $M$ is a triangulated $(d-1)$-manifold on $n$ vertices (allowing for non-orientability and boundary), then

$$
\begin{aligned}
\left(\begin{array}{l}
d \\
j
\end{array}\right) \mathrm{b}_{j-1}(M)+\left(\begin{array}{c}
d \\
j-1
\end{array}\right) \mathrm{b}_{d-j}(M) \leq\left(\begin{array}{c}
n-d+j-1 \\
j
\end{array}\right) & \\
& \text { for } 1 \leq j \leq \frac{d}{2} .
\end{aligned}
$$

This bound in general is not so good for interesting manifolds, as it seems insensitive to any interesting multiplicative structure in the cohomology ring, let alone homotopy.

Sounds like we know a lot, right? Unfortunately, these bounds are far from tight. Just consider some of the manifolds we learn about first in a topology course:

- Triangulate $\mathbb{R} \mathbb{P}^{n}$. The observation of Arnoux and Marin is best, really, and gives a lower bound of quadratic size in $n$. But triangulations that small are hard to come by. For a long time, the best construction was exponential in $n$, though we at least broke through that barrier recently [AAK20].

- Triangulate the torus $\left(S^{1}\right)^{n}$. The smallest known triangulation, due to Kühnel and Lassman [KL88], needs $2^{n+1}-1$ vertices, and it seems challenging to construct smaller examples. Indeed, as far as we know, this number may even be tight. One is tempted to compare this to a systolic inequality, though I do not know how to make this connection sufficiently precise.
A problem here is that we just do not have good geometric invariants that tell us meaningful answers about the combinatorial size of a complex. The other issue is that constructions in a combinatorial setting are hard to come by, especially if they are to be the small(est), in some sense.

One of the hardest constructions I have ever managed to do was for polytopes with "small" moduli space, that is, where the moduli space of a polytope is the space of polytopes with the same combinatorics, modulo projective transformations. The smallest possible here is a point, and with Ziegler I managed to construct infinitely many polytopes whose moduli space is a point in dimension 69 [AZ15]. Many difficult ideas usually go into these constructions, such as partial differential relations in the former, and for instance probablistic techniques in my possibly favourite example, the counterexample to the extension space conjecture by Gaku Liu [Liu20]. But several related problems remain open, for instance:

Problem 7.1. Are there infinitely many combinatorially distinct types of polytopes of dimension 4 whose moduli space is a point?

You cannot hope to do the same for three-dimensional polytopes, where the number is finite due to Steinitz' theorem [Ste22]. It implies that the space of realisations of a polytope is of dimension of the number of edges of the polytope plus 6 (if we ignore projective transformations), so polytopes we look for can have at most nine edges. The number of such polytopes is finite (hint: it is four.)

Let me give an example coming from topology that has puzzled me recently.

Consider the following problem: I task you to give me many simplicial complexes. To make it simple, let them only be of dimension 2. I give you $n$ triangles to build them with, and you are asked to make them combinatorially distinct.

Can you make superexponentially many?

$\mathrm{Ok}$, that is actually easy. Let us make it more interesting. Every vertex should only be incident to a bounded, say 1000 , number of triangles.

Still, you can construct examples: simply construct a long strip of triangles, and attach some handles.

Ok, final restriction. Please make it contractible.

Problem 7.2. Is the number of contractible complexes with a given number of triangles $n$ and a uniform bound on the vertex degree exponential, or superexponential? What happens if I only restrict to complexes with vanishing reduced homology?

Note that any family of complexes one constructs cannot be too simple. For example, it follows from [BZ11] that collapsible complexes are not enough, and neither are complexes whose Andrews-Curtis complexity, that is, the number of Nielsen operations to reshape it into the trivial presentation, is bounded. It seems that "simply connected" is the real obstruction here, and not contractible. So one could equally ask the question and demand that only the first homotopy group or homology group should vanish.

\section{Geometry of polyhedra}

On the other hand, constructions are not everything. We have to understand the other direction, the restrictions geometry 
imposes, better as well. Let me begin by asking a question which is rather famous in combinatorics:

Problem 8.1. Given a triangulation of a connected d-manifold on $n$ vertices, how many steps does one need from any facet to any other facet?

Here, steps that are allowed are to go from one $d$-simplex to the next via a $(d-1)$-simplex they share. The main question here: Is this diameter a polynomial in $n$ and $d$ ? The answer, so far, is not known. This of course is a version of the polynomial Hirsch conjecture, and related to the question of how long the simplex algorithm could take in the worst case.

What is known is that under geometric restrictions, this diameter is well behaved. For instance, assume that the triangulation satisfies Gromov's no- $\Delta$ condition [Gro87]. Then the natural spherical metric is CAT(1), that is, its sectional curvature is bounded above by 1 in the Alexandrov sense. This means neighbourhoods of vertices are convex by a classical result in geometric group theory. Following a shortest path between any two facets gives a diameter of $n-d+1$ [AB14].

This suggests a stronger relationship between geometric notions of polyhedra and differential geometry that needs to be explored, but for now, we have only very special cases where this is achieved, usually in the form of rather strong comparison theorems.

\section{$9 \quad p$-curvatures and small intervals}

It is tempting to think that every metric sphere has to have a point of positive curvature. This is indeed true if one considers sectional curvature (this is the classical Cartan-Hadamard theorem). On the other hand, Lohkamp proved that spheres and other manifolds of dimension at least 3 admit metrics of negative Ricci curvature [Loh94]. Here is a question that sits in between.

Problem 9.1. Is it true that for every $\ell$, there exists a $k=k(\ell)$ such that every $d$-polytope (or polyhedral sphere), $d \geq k$, has a Boolean interval of length $\ell$ in its face lattice?

The closest geometric analogue is a question asking for obstructions on Gromov's p-curvature, rather than the obstructions to curvature bounds studied classically, leading to a tempting Ramsey-like geometric problem.

Problem 9.2 (Adiprasito-Kalai 2015). Is it true that for every $p \geq 2$ there exists a $k=k(p)$, such that every Riemannian metric $R$ on $S^{d}, d \geq k$ has a point $x$ and a p-dimensional subspace $M$ of $T_{x} R$, such that the average over sectional curvatures at 2-sections in $M$ is non-negative?

Temptingly, the answer to the second problem is for conjectures to be "no way", especially with the experience taught by differential geometry. The first problem (which seems to be due to Perles originally), on the other hand, is conjectured to have a positive answer at least, and is known to have such an answer for small $\ell$, see [KKM00].

\section{Stoker conjecture and comparison theorems for mean curvature}

Let us close (and close the circle), address a question by Mikhail Gromov, and highlight it. In a recent series of lectures on scalar curvature and comparison theorems, [Gro19] put forward the following question concerning polytopes (though I heard it independently from Arseniy Akopyan):

Conjecture 10.1. Assume $P$ and $Q$ are combinatorially equivalent polytopes, such that the dihedral angles of $P$ are bigger or equal than the corresponding dihedral angles of $Q$. Then the polytopes are normally equivalent, that is, their normal fans are related by rotation and reflection.

It should be noted that the problem is easy for polygons, and that it is a simple exercise for simple polytopes (i.e. $d$ dimensional polytopes in which every facet is incident to at most $d$ edges).

It is also true infinitesimally, that is, any infinitesimal deformation of a polytope that does not increase any dihedral angle that does not increase any dihedral angle must leave them constant, and can be continued to a normal equivalence. This follows in various ways: by the Schläfli formula [SS03], the angles must stay constant. According to Weiss' work [Wei05], the desired normal equivalence follows from here.

The alternative is to dualise, to take us back and return to the Lefschetz properties we started with: Consider the normal fan of $P$. The problem then turns to asking whether the geodesic arcs cut by 2-dimensional cones inside the sphere can be infinitesimally deformed without elongating any one of them. It follows from the Hodge-Riemann relations in Section 4 that this cannot happen unless the deformation gives an isometry of the sphere. Indeed, applying them for $k=1$ shows that their length is given as a local minimum of an optimisation problem.

\section{Bibliography}

[Adi18] Karim Adiprasito, Combinatorial Lefschetz theorems beyond positivity, 2018, arXiv:1812.10454.

[Adi19] Karim Adiprasito, $F A Q$ on the g-theorem and the hard Lefschetz theorem for face rings, Rend. Mat. Appl., VII. Ser. 40 (2019), no. 2, 97-111.

[AAK20] Karim Adiprasito, Sergey Avvakumov, and Roman Karasev, A subexponential size $\mathbb{R P}^{n}, 2020$, arXiv:2009.02703.

[AB14] Karim Adiprasito and B. Benedetti, The Hirsch conjecture holds for normal flag complexes, Math. Oper. Res. 39 (2014), no. 4, 1340-1348.

[AHK18] Karim Adiprasito, June Huh, and Eric Katz, Hodge theory for combinatorial geometries, Ann. Math. (2) 188 (2018), no. 2, 381-452.

[AY20] Karim Adiprasito and Geva Yashfe, The Partition Complex: An invitation to combinatorial commutative algebra, 2020, Surveys in Combinatorics, British Combinatorial Committee, arXiv:2008.01044.

[AZ15] Karim Adiprasito and G. M. Ziegler, Many projectively unique polytopes, Invent. Math. 199 (2015), no. 3, 581-652. 
[Ale03] Semyon Alesker, Hard Lefschetz theorem for valuations, complex integral geometry, and unitarily invariant valuations, J. Differ. Geom. 63 (2003), no. 1, 63-95.

[Alo86] Noga Alon, The number of polytopes, configurations and real matroids, Mathematika 33 (1986), 62-71.

[AM91] Pierre Arnoux and Alexis Marin, The Kühnel triangulation of the complex projective plane from the view point of complex crystallography. II, Mem. Fac. Sci. Kyushu Univ. Ser. A 45 (1991), no. 2, 167-244.

[BZ11] Bruno Benedetti and Günter M. Ziegler, On locally constructible spheres and balls, Acta Math. 206 (2011), no. 2, 205-243.

[Bin83] R. H. Bing, The geometric topology of 3-manifolds, vol. 40, American Mathematical Society (AMS), Providence, RI, 1983.

[BP15] Victor M. Buchstaber and Taras E. Panov, Toric topology, vol. 204, Providence, RI: American Mathematical Society (AMS), 2015.

[CDMW18] Gregory R. Chambers, Dominic Dotterrer, Fedor Manin, and Shmuel Weinberger, Quantitative nullcobordism, J. Am. Math. Soc. 31 (2018), no. 4, 11651203.

[Che86] Jeff Cheeger, A vanishing theorem for piecewise constant curvature spaces, Curvature and topology of Riemannian manifolds, Proc. 17th Int. Taniguchi Symp., Katata/Jap. 1985, Lect. Notes Math. 1201, 33-40., 1986.

[CLOT03] Octav Cornea, Gregory Lupton, John Oprea, and Daniel Tanré, Lusternik-Schnirelmann category, Mathematical Surveys and Monographs, vol. 103, American Mathematical Society, Providence, RI, 2003.

[Dod79] Jozef Dodziuk, $l^{2}$ harmonic forms on rotationally symmetric Riemannian manifolds, Proceedings of the American Mathematical Society 77 (1979), no. 3, 395-400.

[EW14] Ben Elias and Geordie Williamson, The Hodge theory of Soergel bimodules, Ann. Math. (2) 180 (2014), no. 3, 1089-1136.

[GM92] Israel Gel'fand and Robert MacPherson, A combinatorial formula for the Pontrjagin classes, Bull. Amer. Math. Soc. (N.S.) 26 (1992), no. 2, 304-309.

[GP86] Jacob Eli Goodman and Richard Pollack, There are asymptotically far fewer polytopes than we thought, Bull. Amer. Math. Soc. (N.S.) 14 (1986), no. 1, 127129.

[Gro82] Mikhael Gromov, Volume and bounded cohomology, Publications Mathématiques de l'IHÉS 56 (1982), 599 (en). MR 84h:53053

[Gro87] Mikhail Gromov, Hyperbolic groups, Essays in group theory, Math. Sci. Res. Inst. Publ., vol. 8, Springer, New York, 1987, pp. 75-263.

[Gro19] Mikhail Gromov, Four lectures on scalar curvature, 2019, preprint, arXiv:1908.10612.

[Grü03] B. Grünbaum, Convex Polytopes, second ed., Graduate Texts in Mathematics, vol. 221, Springer, New York, 2003, update of the 1967 edition.

[Gur02] Leonid Gurvits, Quantum matching theory, 2002, arXiv:0201022.

[Hal35] Philip Hall, On representatives of subsets, J. Lond. Math. Soc. 10 (1935), 26-30.

[Ka191] Gil Kalai, The diameter of graphs of convex polytopes and f-vector theory, Applied geometry and discrete mathematics, DIMACS Ser. Discrete Math. Theoret. Comput. Sci., vol. 4, Amer. Math. Soc., Providence, RI, 1991, pp. 387-411.
[KKM00] Gil Kalai, P. Kleinschmidt, and G. Meisinger, Flag numbers and flagtool, Polytopes - Combinatorics and Computation (Gil Kalai and Günter M. Ziegler, eds.), Oberwolfach Seminars, vol. 29, Birkhäuser Basel, 2000, pp. 75-103.

[KL88] W. Kühnel and G. Lassmann, Combinatorial d-tori with a large symmetry group, Discrete Comput. Geom. 3 (1988), no. 1-2, 169-176.

[Laz04] Robert Lazarsfeld, Positivity in algebraic geometry I: Classical setting: Line bundles and linear series. II: Positivity for vector bundles, and multiplier ideals., Berlin: Springer, 2004.

[Liu20] Gaku Liu, A counterexample to the extension space conjecture for realizable oriented matroids, J. Lond. Math. Soc., II. Ser. 101 (2020), no. 1, 175-193.

[Loh94] Joachim Lohkamp, Metrics of negative Ricci curvature, Ann. of Math. (2) 140 (1994), 655-683.

[Lov19] Shachar Lovett, The analytic rank of tensors and its applications, Discrete Anal. 2019 (2019), 10, Id/No 7.

[McM96] Peter McMullen, Weights on polytopes, Discrete Comput. Geom. 15 (1996), no. 4, 363-388.

[Mur15] Satoshi Murai, Tight combinatorial manifolds and graded Betti numbers, Collect. Math. 66 (2015), no. 3, 367-386.

[Nev07] Eran Nevo, Higher minors and Van Kampen's obstruction, Math. Scand. 101 (2007), no. 2, 161-176.

[Par18] Salman Parsa, On the links of vertices in simplicial $d$-complexes embeddable in the Euclidean $2 d$-space, Discrete Comput. Geom. 59 (2018), no. 3, 663-679.

[SS03] Jean-Marc Schlenker and Rabah Souam, Higher Schläfli formulas and applications, Compos. Math. 135 (2003), no. 1, 1-24.

[Sta87] Richard Stanley, Generalized H-vectors, intersection cohomology of toric varieties, and related results, Commutative algebra and combinatorics (Kyoto, 1985), Adv. Stud. Pure Math., vol. 11, NorthHolland, Amsterdam, 1987, pp. 187-213.

[Ste22] E. Steinitz, Polyeder und Raumeinteilungen, Encyklopädie der mathematischen Wissenschaften, Dritter Band: Geometrie, III.1.2., Heft 9, Kapitel III A B 12 (W. Fr. Meyer and H. Mohrmann, eds.), B. G. Teubner, Leipzig, 1922, pp. 1-139.

[Ven17] Akshay Venkatesh, Cohomology of arithmetic groups and periods of automorphic forms, Jpn. J. Math. 12 (2017), no. 1, 1-32.

[Wei05] Hartmut Weiss, Local rigidity of 3-dimensional conemanifolds, J. Differ. Geom. 71 (2005), no. 3, 437506.

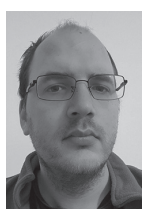

Karim Alexander Adiprasito [karim.adiprasito@ mail.huji.ac.il] is a mathematician at the University of Copenhagen and the Hebrew University of Jerusalem who works in combinatorics, and its connections to geometry and algebra. He is primarily interested in geometric qualities of simplicial complexes and polytopes, and finding combinatorial analogues of classical algebraic and geometric techniques. 